\title{
Mucinose papulosa associada ao hipotireoidismo
}

\author{
Papular mucinosis associated with hypothyroidism
}

\author{
Morgana Boeno Volpato ${ }^{1}$ \\ Martha Pisani Proença ${ }^{3}$ \\ Maria de Fátima Guimarães Scotelaro Alves ${ }^{5}$
}

\author{
Tatiana Jerez Jaime ${ }^{2}$ \\ Alexandre Carlos Gripp ${ }^{4}$
}

Resumo: A mucinose papulosa ou líquen mixedematoso é doença idiopática, manifestada por pápulas, nódulos ou placas por depósito de mucina na derme, na ausência de doença tireoidiana. Apresentamos um caso atípico, com lesões exuberantes em placas, associado ao hipotireoidismo subclínico. Nas mucinoses cutâneas há quantidade anormal de mucina na pele. Tradicionalmente as classificações consideram critério diagnóstico para mucinose papulosa a ausência de doença tireoidiana. Poucos casos associados ao hipotireoidismo são descritos e, assim como o nosso, constituem líquen mixedematoso atípico, sugerindo que a presença de doença tireoidiana não deva ser critério de exclusão para essa doença.

Palavras-chave: Escleromixedema; Hipotireoidismo; Mucinas

\begin{abstract}
Papular mucinosis or lichen myxedematosus is an idiopathic disorder characterized by papules, nodules or plaques caused by mucin deposition in the dermis, in the absence of thyroid disease. The present report describes an atypical case with exuberant lesions in the form of plaques, associated with subclinical hypothyroidism. In cutaneous mucinosis, there is an abnormal quantity of mucin in the dermis. In traditional classifications of papular mucinosis, an absence of thyroid disease constitutes a diagnostic criterion. A few cases of the disease have been reported in association with hypothyroidism constituting, as in the present case, atypical lichen myxedematosus. It is therefore suggested that the presence of thyroid disease should not be considered an exclusion criterion for the diagnosis of this condition.

Keywords: Hypothyroidism; Mucins; Scleromyxedema
\end{abstract}

\section{INTRODUÇÃO}

A mucinose papulosa ou líquen mixedematoso é doença crônica idiopática, caracterizada por pápulas, nódulos ou placas liquenoides causadas por depósito de mucina na derme e grau variável de fibrose, na ausência de doença tireoidiana. ${ }^{1,2}$ Inclui dois subtipos clinicopatológicos. O chamado escleromixedema é forma generalizada e esclerodermoide, acompanhada de gamopatia monoclonal e manifestações sistêmi- cas, que pode ter êxito letal. Por outro lado, a forma papular localizada, que abrange uma série de quadros mais limitados, costuma apresentar curso benigno e crônico. ${ }^{3}$ Ocasionalmente pode ocorrer sobreposição entre diferentes formas da doença ou ainda casos com características únicas e distintas das descritas para todos os subtipos. Estes são os denominados casos atípicos. ${ }^{2,3}$ A classificação das mucinoses cutâ-

Recebido em 08.08.2008.

Aprovado pelo Conselho Consultivo e aceito para publicação em 30.10.08

Trabalho realizado no serviço de dermatologia do Hospital Universitário Pedro Ernesto da Universidade do Estado do Rio de Janeiro (Uerj) - Rio de Janeiro (RJ), Brasil Conflito de interesse: Nenhum / Conflict of interest: None

Suporte financeiro: Nenhum / Financial funding: None

Médica pós-graduanda em dermatologia pela Universidade do Estado do Rio de Janeiro (Uerj) - Rio de Janeiro (RJ), Brasil. Médica pós-graduanda em dermatologia pela Universidade do Estado do Rio de Janeiro (Uerj) - Rio de Janeiro (RJ), Brasil.

Médica pós-graduanda em dermatologia pela Universidade do Estado do Rio de Janeiro (Uerj) - Rio de Janeiro (RJ), Brasil.

Professor assistente do serviço de dermatologia da Universidade do Estado do Rio de Janeiro (Uerj), mestre em dermatologia pela Universidade Federal

Fluminense (UFF) - Rio de Janeiro (RJ), Brasil.

Professora adjunta do serviço de dermatologia da Universidade do Estado do Rio de Janeiro (Uerj), doutora em dermatologia pela Universidade Federal do Rio de Janeiro (UFRJ) - Rio de Janeiro (RJ), Brasil. 
neas é baseada em critérios que incluem o aspecto clínico das lesões de pele, suas características histopatológicas e associação ou não com alterações sistêmicas. De acordo com a mais recente classificação, a ausência de doença da tireoide é um critério diagnóstico para o líquen mixedematoso. Porém, a classificação das mucinoses cutâneas é bastante complexa e ainda controversa. ${ }^{3,4}$ Relatamos um caso de mucinose papulosa atípica, com apresentação exuberante em placas na presença de hipotireoidismo subclínico sintomático.

\section{RELATO DO CASO}

Mulher de 55 anos, procedente do Rio de Janeiro, procurou nosso serviço com história de cerca de seis anos de placas eritêmato-amareladas e assintomáticas no dorso superior. Referiu que as lesões eram menores e há cerca de dois anos tornaram-se infiltradas e mais eritematosas, e que nos últimos seis meses surgiu nova lesão com bordas elevadas e centro deprimido, da cor da pele, na região frontal. Tinha história médica pregressa de hipotireoidismo, diagnosticado há cerca de seis anos, e relatou ter feito tratamento com levotiroxina, o qual interrompeu por conta própria e manteve-se sem até o presente momento. Além das lesões cutâneas, a paciente queixava-se de fadiga, sonolência, artralgias nas mãos, punhos, cotovelos e ombros. No exame dermatológico apresentava placas eritêmato-amareladas, infiltradas, bem delimitadas, arredondadas e com tamanho variando de 4 a $6 \mathrm{~cm}$ no maior diâmetro, distribuídas difusamente no dorso superior (Figuras 1 e 2). Havia outra lesão, cor da pele, anular, com borda elevada e depressão central, medindo cerca de $1,5 \mathrm{~cm}$ de diâmetro, na região frontal (Figura 3). A biópsia de lesão do dorso eviden-

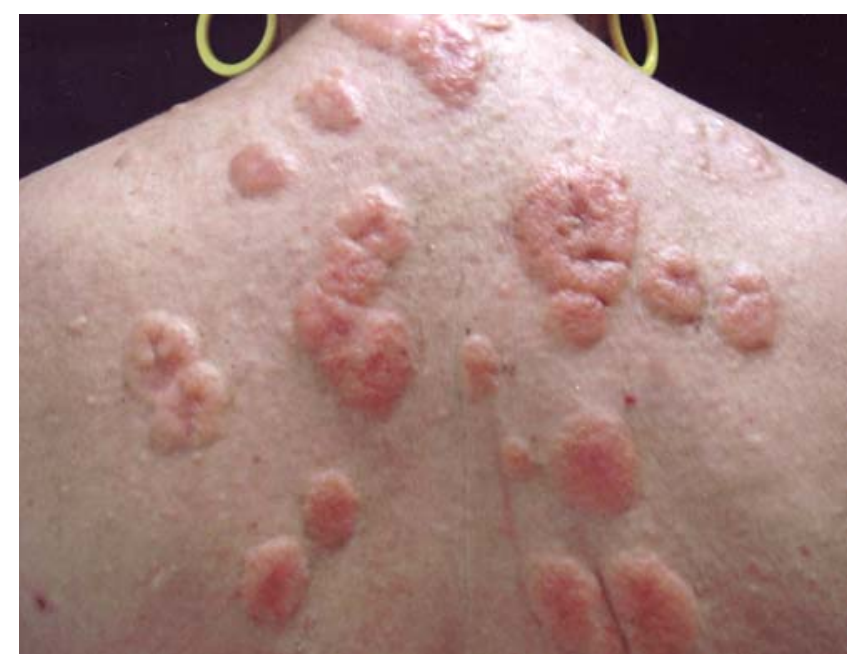

Figura 1: Placas eritêmato-amareladas, infiltradas, bem delimitadas no dorso superior

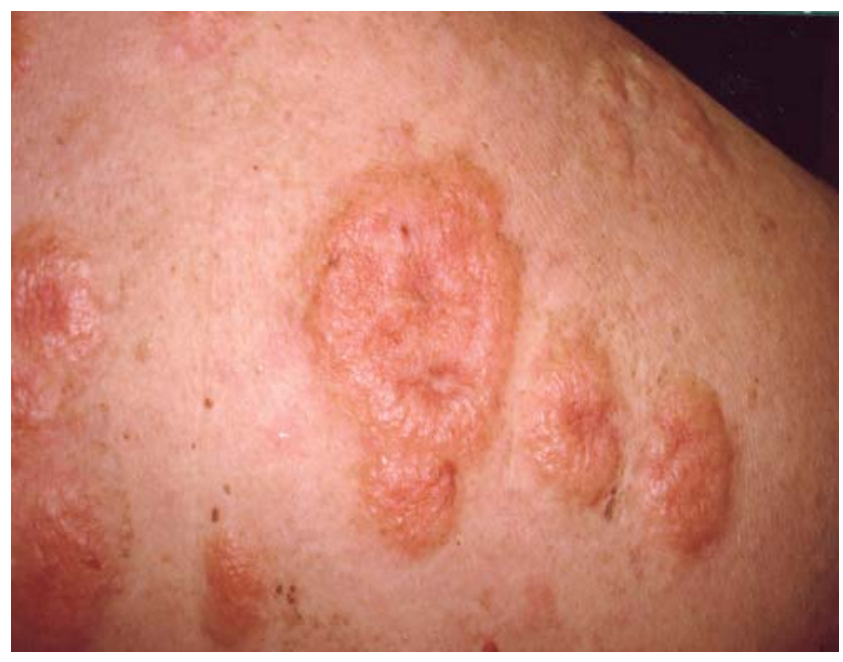

Figura 2: Detalhe das lesões

ciou na derme superior e média intensa dissociação de fibras colágenas com discreto infiltrado inflamatório mononuclear perivascular (Figura 4). A coloração pelo ferro coloidal foi positiva, demonstrando presença de grande quantidade de mucina entre os feixes (Figura 5). O diagnóstico histopatológico foi de mucinose papulosa. Nos exames laboratoriais apresentava TSH 15,45 (normal: 0,27-4,2) e T4 livre 1,02 (normal: 0,93-1,7), sendo diagnosticado hipotireoidismo subclínico sintomático e iniciado tratamento com levotiroxina $50 \mathrm{mcg} / \mathrm{dia}$. Após 15 dias de tratamento a paciente apresentava melhora dos sintomas sistêmicos, sem alterações no quadro cutâneo até então. Os demais exames laboratoriais, como hemograma, função renal, sorologias para HIV, hepatites e eletroforese de proteínas, foram normais, evidencian-

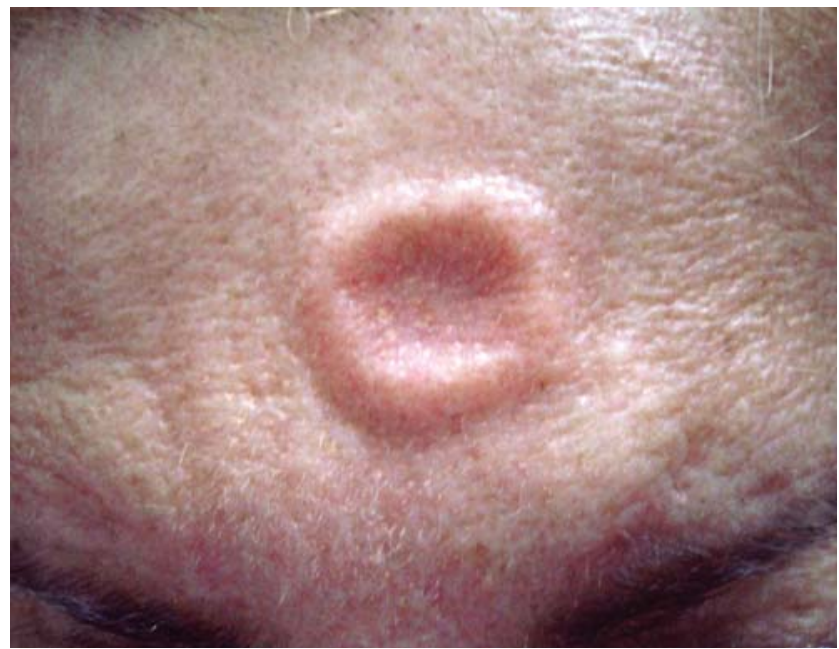

FigurA 3: Lesão cor da pele, anular, com borda elevada e depressão central na região frontal 

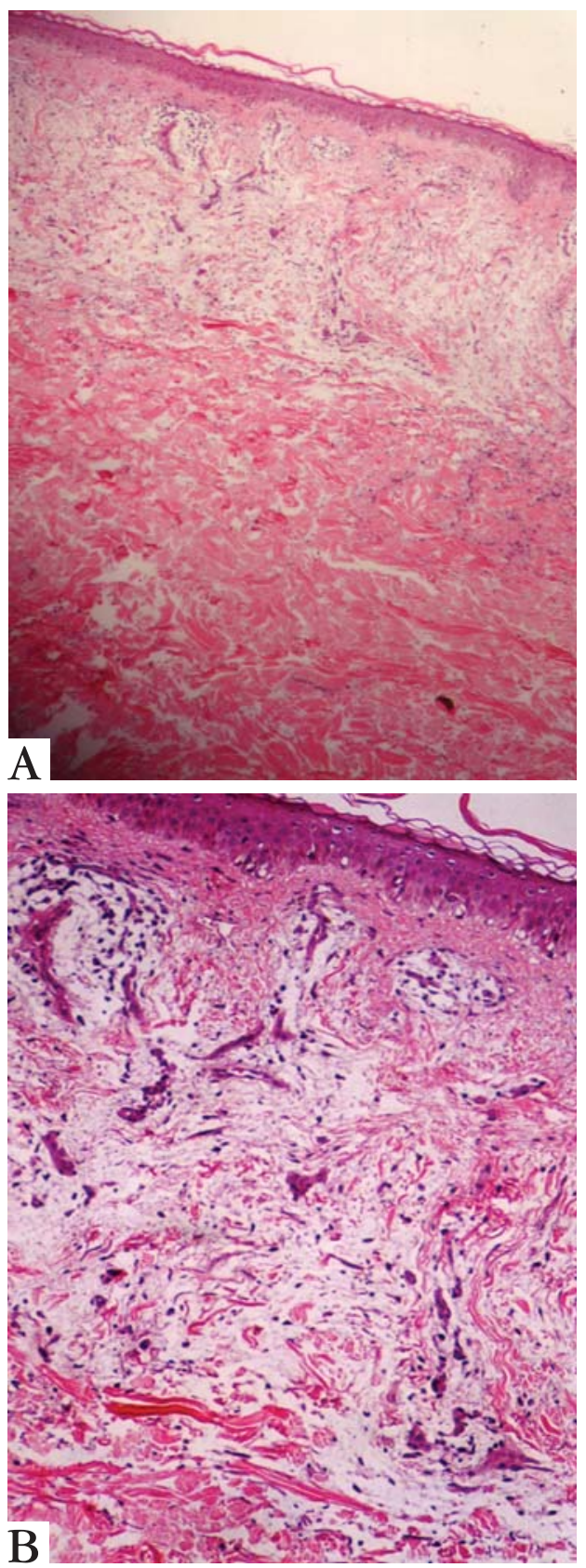

Figura 4: A. Exame histopatológico de lesão do dorso mostrando na derme superior e média intensa dissociação de fibras colágenas com discreto infiltrado inflamatório mononuclear perivascular (HE, 40x); $\mathbf{B}$ Maior aumento (HE, 100x)

do apenas aumento do VHS (48 $\mathrm{mm}$; normal até 10). A paciente foi encaminhada à hematologia, que excluiu a presença de gamopatia monoclonal. Foi também avaliada pela reumatologia, que evidenciou sinovite nas articulações dos punhos, metacarpofalangeanas, interfalangeanas e dos tornozelos, com história de rigidez matinal superior a duas horas.

\section{DISCUSSÃO}

As mucinoses cutâneas são grupo heterogêneo de desordens em que quantidade anormal de mucina é encontrada na pele. ${ }^{2}$ Tradicionalmente são divididas em: primárias, em que o depósito de mucina é carac-

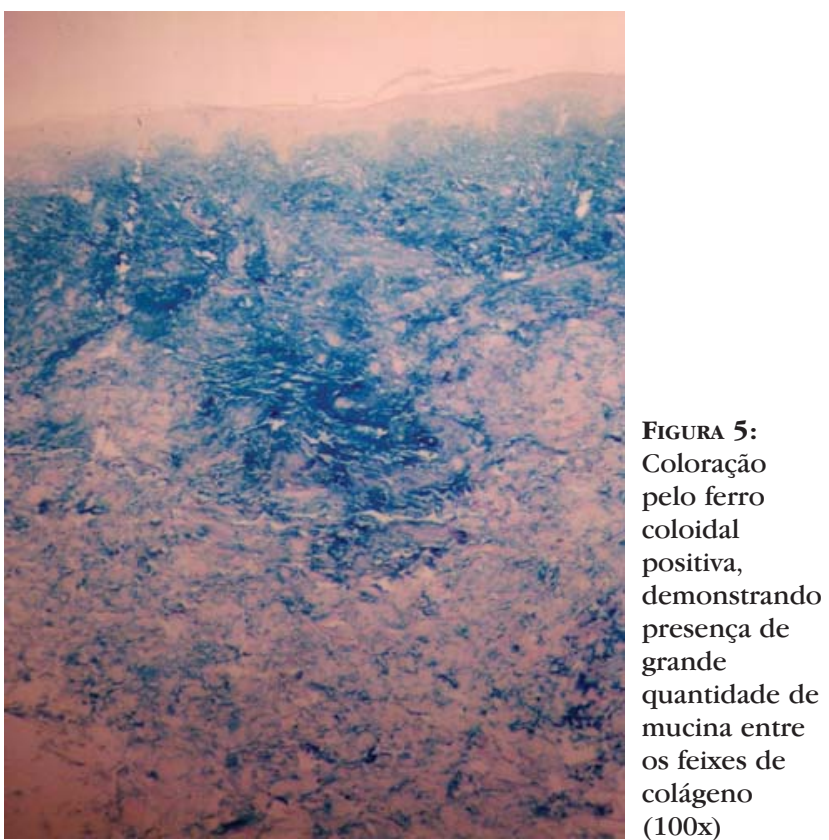

terística histológica principal, e secundárias, em que a mucina é apenas um achado adicional. ${ }^{2}$ Dentre as mucinoses primárias dérmicas encontra-se o líquen mixedematoso ou mucinose papulosa. ${ }^{2} \mathrm{O}$ líquen mixedematoso inclui dois subtipos: uma forma papular generalizada e esclerodermoide (também chamada escleromixedema) com gamopatia monoclonal e manifestações sistêmicas, sem alterações tireoidianas; e uma forma papular localizada. ${ }^{3}$ A forma localizada caracteriza-se por pápulas, nódulos e placas geralmente cor da pele, localizadas nos membros superiores, inferiores e tronco, sem características de esclerose, paraproteinemia, acometimento sistêmico ou doença tireoidiana. ${ }^{3}$ É dividida em cinco subtipos: 1) forma papular discreta; 2) mucinose papular persistente acral; 3) mucinose cutânea com cura espontânea; 4) mucinose cutânea da infância e 5) forma nodular pura. ${ }^{1,3}$ Um terceiro grupo inclui pacientes com líquen mixedematoso com características atípicas ou intermediárias entre as da forma generalizada e da localizada ${ }^{1,2,3}$ As classificações atuais consideram critério diagnóstico para mucinose papulosa a ausência de doença tireoidiana. ${ }^{3}$ Há na literatura relato de três casos associados ao hipotireoidismo, que, assim como o presente relato, constituem casos de líquen mixedematoso atípico. ${ }^{4,5,6} \mathrm{~A}$ razão da formação dos depósitos papulares de mucina no hipotireoidismo não é conhecida. Eles possivelmente representam uma resposta exagerada do tecido local a uma anormalidade generalizada do metabolismo do tecido conectivo induzida pela deficiência no hormônio da tireoide. ${ }^{7}$ Há evidências de que no hipotireoidismo o acúmulo exces- 
sivo de ácido hialurônico na pele e outros tecidos decorre de uma diminuição proporcionalmente maior na sua degradação do que a que ocorre em sua síntese. ${ }^{7}$ Em dois casos previamente relatados, a reposição de hormônio tireoidiano levou a uma melhora da mucinose cutânea, enfatizando a associa- ção entre essas entidades, na qual a mucinose papulosa pode ser vista como uma manifestação dermatológica do hipotireoidismo., ${ }^{5,6}$ Assim como Archibald et al., ${ }^{4}$ acreditamos que a presença de doença tireoidiana não deve ser critério de exclusão para o diagnóstico de líquen mixedematoso.

\section{REFERÊNCIAS}

1. Rongioletti F. Lichen mixedematosus (papular mucinosis): new concepts and perspectives for an old disease. Semin Cutan Med Surg. 2006;25:100-4.

2. Rebora A, Ronglioletti F. Mucinoses. In: Bolognia JL, Jorizzo JL, Rapini RP, editors. Dermatology. Spain: Mosby/Elsevier; 2004. p. 647-58.

3. Rongioletti F, Rebora A. Updated classification of papular mucinosis, lichen myxedematosus, and scleromyxedema. J Am Acad Dermatol. 2001;44:273-81.

4. Archibald GC, Calvert HT. Hypothyroidism and lichen myxoedematosus. Arch Dermatol. 1977;113:684.
5. Schaeffer D, Bruce S, Rosen T. Cutaneous mucinosis associated with thyroid dysfunction. Cutis. 1983;32:449-52,454,456.

6. Martin-Ezquerra G, Sanchez-Regaña M, Massana-Gil J, Umbert-Millet P. Papular mucinosis associated subclinical hypothyroidism: improvement with thyroxine therapy. J Eur Acad Dermatol Venereol. 2006;20:1340-1.

7. Jakubovic HR, Salama SS, Rosenthal D. Multiple cutaneous focal mucinoses with hypothyrodism. Ann Intern Med. 1982;96:56-8.

ENDEREÇO PARA CORRESPONDÊNCIA / MAILING ADDRESS: Morgana Boeno Volpato

Rua 15 de Novembro, 267 99010090 Centro Passo Fundo, RS Tel./Fax: 543311 6688; 33581549 e 96295731 , E-mail: morvolp@gmail.com

Como citar este artigo/How to cite this article: Volpato MB, Jaime TJ, Proença MP, Gripp AC, Alves MFGS. Mucinose papulosa associada ao hipotireoidismo. An Bras Dermatol. 2010;85(1):89-92. 